\title{
Multi-scale Modeling of Electron Beam Melting of Functionally Graded Materials
}

\author{
Wentao Yan ${ }^{\mathrm{a}, \mathrm{b}}$, Wenjun $\mathrm{Ge}^{\mathrm{a}}$, Jacob Smith ${ }^{\mathrm{b}}$, Stephen Lin ${ }^{\mathrm{b}}$, Orion L Kafka ${ }^{\mathrm{b}}$, \\ Feng $\operatorname{Lin}^{\mathrm{a}, \mathrm{c}}$, Wing Kam Liu ${ }^{\mathrm{b}, \mathrm{c}}$ \\ ${ }^{a}$ Department of Mechanical Engineering, Tsinghua University, Beijing, 100084, P.R. \\ China \\ ${ }^{b}$ Department of Mechanical Engineering, Northwestwern University, Evanston, IL 60201, \\ United States \\ ${ }^{c}$ Corresponding authors: linfeng@tsinghua.edu.cn,w-liu@northwestern.edu
}

\begin{abstract}
Electron Beam Melting (EBM) is a promising powder-based metal Additive Manufacturing (AM) technology. This AM technique is opening new avenues for Functionally Graded Materials (FGMs). However, the manufacturing process, which is largely driven by the rapidly evolving temperature field, poses a significant challenge for accurate experimental measurement. In this study, we develop a novel multi-scale heat transfer modeling framework to investigate the EBM process of fabricating FGMs. Our heat source model mechanistically describes heating phenomena based on simulation of micro-scale electron-material interactions. It is capable of accounting for the material properties and electron beam properties that depend on experimental setup. The heat source model is utilized in the thermal evolution model of individual powder particles at the meso-scale to elucidate the melting and coalescing processes for mixed powder particles of different materials and different sizes. Another meso-scale simulation is conducted to evaluate the effective thermal conductivity of the original powder bed for the macro-scale model. A macro-scale heat transfer model is developed, in which the coalescence state is tracked to determine the effective material properties of the powder bed. Predictions of molten pool size are compared against published experimental results for validation.
\end{abstract}

Keywords: Additive Manufacturing, Electron Beam, Functionally Graded Material, Multi-scale Modeling 


\section{Introduction}

Additive Manufacturing (AM) technologies for metallic components, including Selective Laser Melting (SLM), Electron Beam Melting (EBM) and Laser Engineered Net Shaping (LENS), have been drawing increasing attention over the past decade. In addition to manufacturing components with complex geometry, an appealing potential of these powder-based AM technologies is opening new avenues of locally manipulating the chemical compositions and the mechanical properties. For example, Ge, et al. [1][2][3], manufactured functionally graded Ti-TiAl materials using EBM; a process schematic is shown in Fig.1. In this paper, we perform an investigation into the driving mechanisms for the overall fabrication process of Functionally Graded Materials (FGMs).

The EBM process for FGM is very complex; however, the fabrication procedure mainly consists of four repeated steps [1].

1. Apply one layer of powder on a preheated platform. For each layer, we can design and tailor the mixture ratio of the two different types of powder (see Fig. 1), in order to manipulate the chemical compositions. In the experiments, atomized $\mathrm{Ti}-6 \mathrm{Al}-4 \mathrm{~V}$ and $\mathrm{Ti}-47 \mathrm{Al}-2 \mathrm{Cr}-2 \mathrm{Nb}$ powders were used [1].

2. Preheat the powder bed to slightly bond the powder particles. This helps prevent powder scattering.

3. Selectively melt the powder bed using a focused electron beam to form the $2 \mathrm{D}$ cross section of the desired 3D geometry. The beam power and scan speed are key factors which greatly influence the final part quality. Scan strategies, including scan path and repeated scan, are also influential. In Ref. [1], the scan speed and hatch spacing were kept constant at $500 \mathrm{~mm} / \mathrm{s}$ and $0.2 \mathrm{~mm}$, respectively, and the scan was repeated three times with successive beam currents of $2 \mathrm{~mA}, 4 \mathrm{~mA}$ and $6 \mathrm{~mA}$.

4. Move the platform down by one layer thickness. The layer thickness can be adapted depending upon the desired geometry to achieve both high fabrication quality and short build time. In the experiments [1], the layer thickness was fixed at $100 \mu \mathrm{m}$.

The efficacy of fabricating FGMs is largely determined by melting and coalescing of the two different types of powder particles. The temperature field regulates the process. It not only directly determines the melting and 
solidification process but also influences the molten pool flow. However, it is difficult to accurately measure the temperature field in experiments: the peak temperature is very high (up to the boiling temperature of the metal) and the thermal gradient and heating/cooling rates are extremely large (up to $10^{6}$ $\mathrm{K} / \mathrm{m}$ and $10^{6} \mathrm{~K} / \mathrm{s}$ [4], respectively). The fabrication parameters for a qualified functionally graded component are traditionally determined through a long and costly experimental trial-and-error process. Effective numerical heat transfer modeling of the process has become a powerful tool for understanding and optimizing the EBM process [5], thereby reducing the extent of required experimental studies.

The macro-scale heat transfer models for SLM and EBM have been extensively investigated to calculate the temperature field and history, based on which a few more characteristics such as thermal stress [6] and microstructure [4] can be further predicted. A comprehensive review is given by [7]. The basic principle is to treat the powder bed as a reduced-density, low-thermalconductivity continuum material [8].

A few powder-scale models resolving the randomly distributed particles in the powder bed have been developed to investigate the evolution process of individual powder particles [9] [10] [11]. Körner et al. [9] employed the Lattice Boltzmann Method (LBM) to study the successive consolidation process in powder layers. Khairallah et al. [10] developed a meso-scopic model to investigate the formation mechanism of pores, spatter and denudation using the ALE3D multi-physics code. The model incorporated surface tension, Marangoni effect and recoil pressure. However, both of these codes are not as accessible to the AM research community as open-source or commercialized codes, and the computation expense of these models can be up to the order of 100,000 cpuh [12], which is considerably beyond the affordability of most AM research groups. Therefore, while these models provide insight to the physical mechanisms, they are not feasible in a concurrent design sense.

Most of the aforementioned models are focused on single material fabrication processes, and few studies have been done to provide insights of the fabrication process of FGMs. Moreover, most of the electron beam heat source models are either approximated as surface heat flux or assumed to be volumetric energy input similar with the molten pool shape, lacking a strong physical foundation.

In this study, we present a multi-scale modeling framework to understand and optimize the manufacturing process of FGMs at various scales. It consists of a new heat source model derived from the micro-scale electron-material 
interaction simulations, a heat transfer model for individual powder particles at the meso-scale, and a homogeneous macro-scale model. The framework is outlined in Fig.1. The heat source model is material dependent and experimental setup specific. Since it incorporates the physics of the system, this model has obvious application to process design; other models, e.g. those based on molten pool shape, do not. To the best of our knowledge, this is the first multi-scale model specifically for the FGM fabrication process. Moreover, the computations can be done with a typical desktop or laptop making it a reasonable task. This means that the models can be used to rapidly provide fabrication parameter selection guidelines.

Körner et al.[9] demonstrated that the consolidation of powder particles is limited by thermal diffusion rather than molten material flow. These simulations showed that the melted portions of powder particles reshape to coalesce immediately after the liquidus temperature is reached. From this observation, we assume that if the mixed powder particles of different materials reach the liquidus temperature then the chemical compositions evenly mix. This assumption precludes the necessity of capturing molten pool flow and chemical element diffusion, since the details of the mixing process are not directly topical.

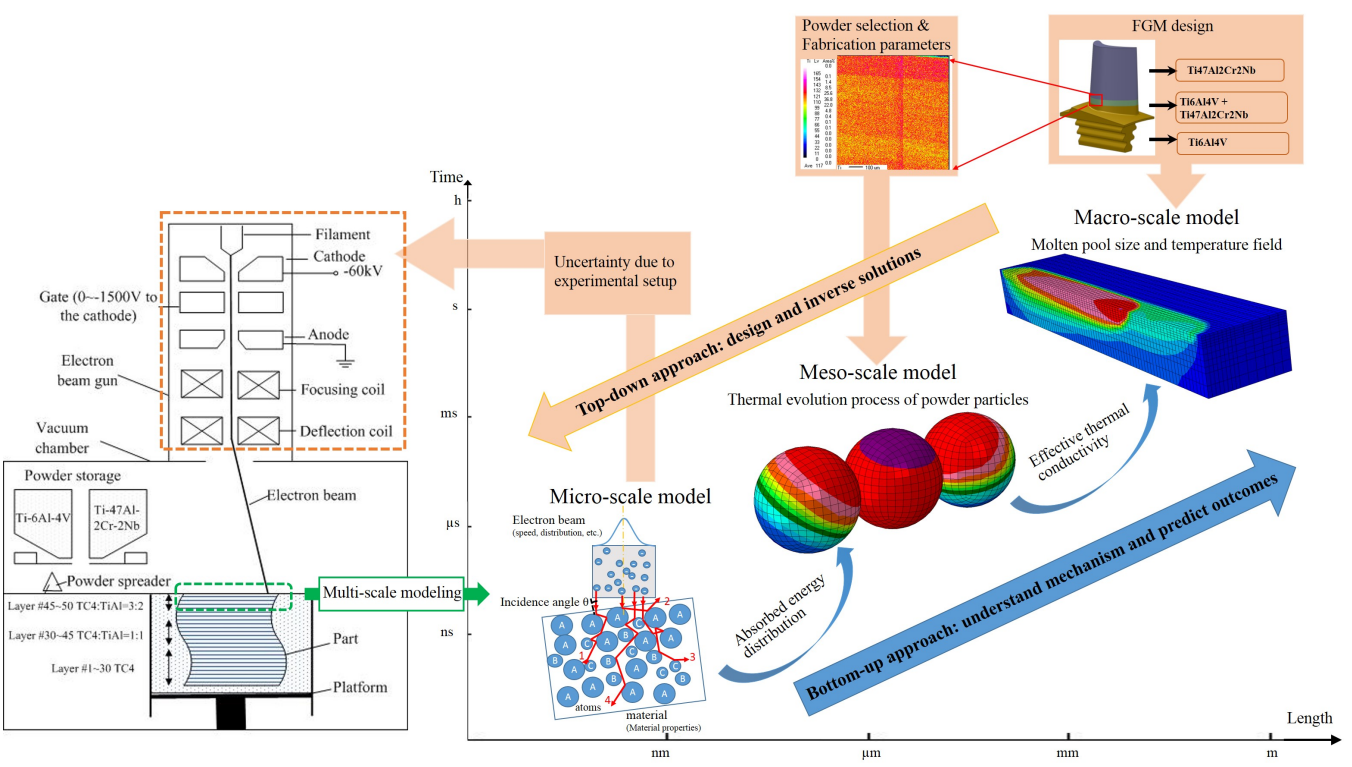

Figure 1: Schematic diagram of proposed multi-scale modeling framework 
In Section 2, we introduce a new, mechanistic heat source model, which is material-dependent and experimental setup specific (accounting for electron beam properties and incidence angle). The heat source model is derived from micro-scale electron-material interaction simulations, which is the driving physical mechanism of electron beam heating. In Section 3, a meso-scale heat transfer model based on the new heat source is used to comprehensively describe the thermal evolution process of individual powder particles of different materials and sizes. Another meso-scale simulation is conducted to evaluate the effective thermal conductivity of the original powder bed for the macro-scale model. In Section 4, a macro-scale heat transfer model is developed using an effective continuum material model to represent the powder bed. The material properties are set to be dependent on the coalescent state rather than the temperature, providing a simple and physically convincing model of the material addition process. In Section 5, the simulation results of the aforementioned models are presented and discussed in detail. Good agreement between simulations and published experimental results [1] is achieved.

\section{Heat source model}

Established based on the Monte Carlo simulations of electron-material interaction at the micro-scale [13], our proposed heat source model is capable of accounting for the material properties, incidence angle and electron beam properties which are dependent on the experimental setup. Thus, the heat source model may be easily applied to process design and provide insight into uncertainty in experiments.

The general formula of the proposed heat source model is

$$
q=Q \times \eta \times F_{\text {pene }} \times F_{\text {section }}
$$

where the five major terms are: the input energy density $q$, the electron beam power $Q$, the energy absorptivity $\eta$, the energy distribution along the incidence direction $F_{\text {pene }}$ and the energy distribution in the cross section of the electron beam $F_{\text {section }}$.

\subsection{Electron beam power $Q$}

The electron beam power $Q$ is

$$
Q=U I
$$


where $U$ is the acceleration voltage and $I$ is the beam current. The acceleration voltage $U$ is determined by the electric potential difference between the cathode and anode, which is usually kept stable at $60 \mathrm{kV}$, as shown in Fig.1. The beam current $I$ is the main control parameter to adjust the power $Q$. The emission current from the filament is easily controlled except when the filaments are first installed or about to fail [14], but the beam current that reaches the specimen is different than the emission current due to the electron loss during the transport from the filament to the specimen. Thus, the beam current to the specimen should be calibrated before experiments to reduce the uncertainty in the electron beam power.

\subsection{Energy absorptivity $\eta$}

The energy absorptivity $\eta$ is determined by the material properties, electron speed and incidence angle (see Table.1). During the electron-material interactions, the energy is either absorbed by the material or lost due to back-scattered electrons, considering as negligible the influence of secondary electrons and emitted X-rays. The energy loss due to back-scattered electrons can be evaluated by multiplying the back-scattering coefficient and the normalized back-scattered energy (see back-scattered energy distribution in the sub-figures on the lower left corners of Fig.2 (d) and (f)). These are calculated directly with the Monte Carlo simulations and are functions of incidence angle, material properties and electron speed. When applied to complex geometries such as spherical powder particles, the heat source model accounts for varying absorption and reflection as a function of incidence angle at different locations through adjusting $\eta$.

\subsection{Energy distribution in the beam cross section $F_{\text {section }}$}

The energy distribution in the beam cross section $F_{\text {section }}$ is proportional to the electron distribution, generally assumed to be a Gaussian distribution given as,

$$
F_{\text {section }}=\frac{N}{\pi R_{b}{ }^{2}} \exp \left(-N \frac{\left(x-x_{s}\right)^{2}+\left(y-y_{s}\right)^{2}}{R_{b}{ }^{2}}\right)
$$

where $x_{s}$ and $y_{s}$ denote the coordinates of the center of the beam. The distribution is characterized by the concentration coefficient of the electron beam $N$ and the defined beam radius $R_{b}$, which means that $1-e^{-N}$ of the total beam power is concentrated within $R_{b}$. For instance, $N=3$ indicates that $95 \%$ of the total beam power is concentrated within $R_{b}$. On the other 
hand, if we define the beam radius $R_{b}$ to be the region containing $99 \%$ of the total power, then $N=4.6$. It should be noted that there is no universally accepted defined beam radius [14]. The coefficient $\left(\frac{N}{\pi R_{b}{ }^{2}}\right)$ is used to enforce conservation of energy: that is to make the integral over the cross section be 1, $\int_{S} F_{\text {section }} d S=1$.

The assumed Gaussian electron distribution lacks a strong physical foundation. Electron distributions in the beam cross section is largely determined by the filament, focusing coil and the state of the deflection coil. It should be emphasized that the filaments and focusing coils differ and may also change with time due to heating, oxidation and vaporization. Another important factor is the vacuum. Lower vacuum may lead to a larger electron beam radius. Therefore, the filament, focusing coil and vacuum are the primary sources of uncertainty in the heat source model as they have the strongest influence over the beam radius. However, our proposed model can incorporate these uncertainties as long as accurate measurements of the beam radius are provided, which is currently a challenging task in itself.

\subsection{Energy distribution along the incidence direction $F_{\text {pene }}$}

The energy distribution along the incidence direction $z$ is established based on the Monte Carlo simulations (see Fig.2 (d) and (e)). The formula is given as

$$
F_{\text {pene }}=\frac{1}{\delta \int_{-\frac{z_{0}}{\delta}}^{+\infty} \exp \left(-t^{2}\right) d t} \exp \left(-\frac{\left(z-z_{T}(x, y)-z_{0}\right)^{2}}{\delta^{2}}\right)
$$

where $z_{0}, \delta$ and $z_{T}(x, y)$ are the location with the highest energy density, the characteristic penetration depth, and the $z$ coordinate of the material surface, respectively. The parameters $z_{0}$ and $\delta$ are obtained from a curve fit to the simulated energy distribution in the $z$ direction [13]. The coefficient $\left(\frac{1}{\delta \int_{-\frac{z_{0}}{\delta}}^{+\infty} \exp \left(-t^{2}\right) d t}\right)$ is again to ensure conservation of energy: the integral along the incidence direction must be $1, \int_{z} F_{\text {pene }} d z=1$.

The parameters $z_{0}$ and $\delta$ are physically determined by the material properties and acceleration voltage (see Table 1). Since most equipment sets the acceleration voltage as $60 \mathrm{kV}$, the energy distribution along the incidence direction is quite similar for a given material for these equipment. Thus, uncertainty of this term is minor for a given material. 


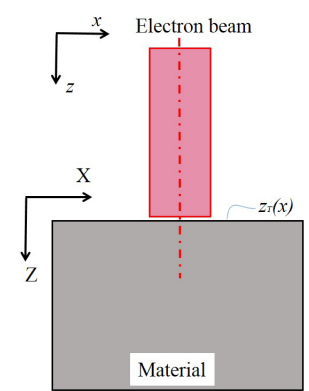

(a)

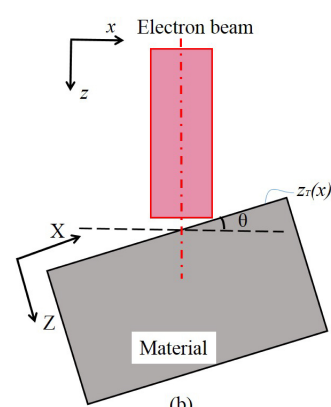

(b)

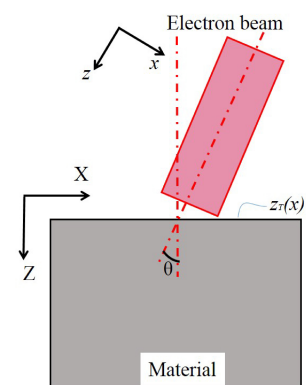

(c)
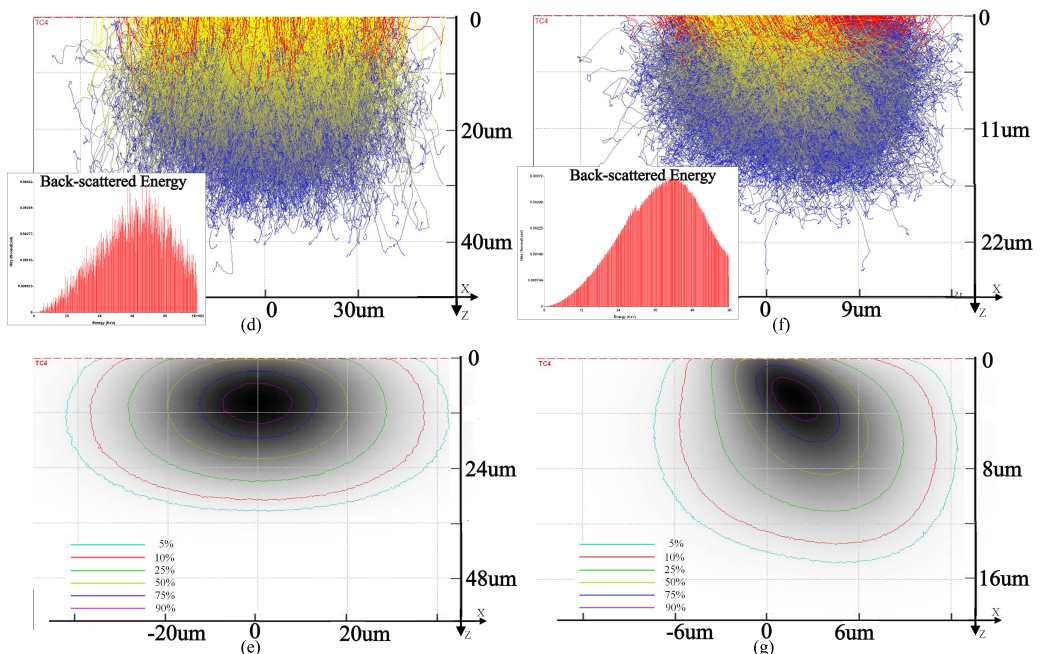

Figure 2: The proposed heat source model is able to account for different electron beam incidences including: (a) normal beam, (b) tilted substrate, and (c) deflected beam. The coordinate system for the electron beam is $x z$, and $X Z$ is for the material. Electron trajectories and absorbed energy distribution are simulated for two various incidence angles and electron beam properties: normal incidence under $100 \mathrm{kV}$ in (d) and (e), and tilted incidence under $60 \mathrm{kV}$ in (f) and (g). The red trajectories represent back-scattered electrons, the other trajectories are yellow when the energy of the electron is high, and then turn blue once the energy becomes lower than a specific value. The color line with a percentage represents the percentage of energy absorbed by the area outside the line.

Table 1: Calculated $z_{0}, \delta$ and $\eta$ for different materials and acceleration voltages

\begin{tabular}{llll}
\hline & $z_{0}(\mu \mathrm{m})$ & $\delta(\mu \mathrm{m})$ & $\eta$ for a normal incidence \\
\hline Ti-6Al-4V, 60kV & 4.4 & 5.7 & 0.895 \\
Ti-6Al-4V, 100kV & 10.9 & 13.9 & 0.905 \\
Ti-47Al-2Cr-2Nb, 60kV & 5.0 & 6.7 & 0.891 \\
\hline
\end{tabular}




\subsection{Effect of the incidence angle}

The effect of the incident angle requires attention when applying the heat source model. Specifically, the material surface may be tilted (see Fig.2 (b)) or the electron beam may be deflected (see Fig.2 (c)). These two cases are in essence the same. The heat source model can be transformed into the material coordinate system by simply rotating the coordinate axis $x$ and $z$ by the incidence angle $\theta$. The coordinate transformation is,

$$
\left\{\begin{array}{l}
x=X \cos \theta-Z \sin \theta \\
z=X \sin \theta+Z \cos \theta
\end{array}\right.
$$

For the example shown in Fig.2 (c), we calculate the coordinates with the highest energy density in the material coordinate system to be $X_{0}=$ $X_{s}+z_{0} \sin \theta$ and $Z_{0}=Z_{s}+z_{0} \cos \theta$. This is demonstrated by the simulation result in Fig.2 (g). The material coordinates with the highest energy density are $X_{0}=2.0 \mu \mathrm{m}=4.4 \mu \mathrm{m} \times \sin 30^{\circ}$ and $Z_{0}=3.9 \mu \mathrm{m}=4.4 \mu \mathrm{m} \times \cos 30^{\circ}$.

Since the deflection angle of the electron beam is usually controlled in a small range, a titled surface is the most common case, especially for complex surface shapes. We need to have the surface coordinate function $z_{T}(x, y)$ and adjust the energy absorptivity $\eta$ according to the incidence angle. For instance, when a vertical electron beam (acceleration voltage is $60 \mathrm{kV}$ ) is incident at a spherical powder particle of Ti-6Al-4V, the surface coordinate function of a spherical powder particle is given as,

$$
z_{T}(x, y)=z_{c}+\sqrt{R^{2}-\left(x-x_{c}\right)^{2}-\left(y-y_{c}\right)^{2}}
$$

where $x_{c}, y_{c}, z_{c}$ and $R$ denote the center coordinates and the radius of the spherical powder particle, respectively. The incidence angle is dependent on the specific position on the spherical surface, as is the energy absorptivity $\eta$. For a normal incidence at the sphere center $\left(x=x_{c}\right) \eta$ is 0.895 , while $\eta$ is 0.874 at an incidence angle of $30^{\circ}$ (where $\sqrt{\left(x-x_{c}\right)^{2}+\left(y-y_{c}\right)^{2}}=R / 2$ ). Near the edge of the sphere, $\eta$ approaches 0 . Therefore, the effective absorptivity for a spherical particle is approximately 0.6, which is employed in the macro-scale simulations. We can substitute Eq.6 into Eq.3, and then into Eq.1 to obtain the heat source model for a spherical powder particle. 


\section{Meso-scale powder particle model}

\subsection{Thermal evolution model of powder particles}

The thermal evolution of spherical powder particles of Ti-6Al-4V and Ti47Al-2Cr-2Nb, schematically shown in Fig.3 (a), was simulated when heated by an electron beam moving at the speed of $500 \mathrm{~mm} / \mathrm{s}$ with the currents of $2 \mathrm{~mA}(120 \mathrm{~W}), 4 \mathrm{~mA}(240 \mathrm{~W})$ and $6 \mathrm{~mA}(360 \mathrm{~W})$, respectively. The effect of particle size on the melting process was also investigated by running the simulation case schematically shown in Fig.3 (b). Since the thermal conductivity between powder particles and the base plate is low (illustrated in Section 3.2), the influence of the base plate can be neglected, especially at a time scale shorter than $1 \mathrm{~ms}$. Thus, the base plate was not considered in the meso-scale models.

Since the hatch spacing is $200 \mu \mathrm{m}$ and the diameter of the electron beam is $350 \mu \mathrm{m}$, the powder particles located in the middle of two neighboring scan lines are least likely to get melted neglecting the heat accumulated during previous scans. Thus, the complete melting of powder particles between scan lines largely determines the success of FGM fabrication. To reflect this, particles are placed $100 \mu \mathrm{m}$ away from the beam center in the models shown in Fig.3 (a) and (b).

The material properties for Ti-6Al-4V and Ti-47Al-2Cr-2Nb are listed in Table.2, as presented by [15] and [16]. In order to compensate the missing convective heat transfer due to the molten material flow, the thermal conductivity at the temperature above the liquidus temperature was artificially enlarged by a factor of 10 in the following models. The factor is selected based on the reshaping time scale, which is about one order of magnitude smaller than the thermal diffusion time scale [9]. Thus, the effective thermal diffusion in the molten regions caused by the reshaping flow is approximately to 10 times of the pure thermal diffusion. It should also be mentioned that neither evaporation nor the subsequent mass loss was considered in this study, since evaporation is not the concern of this study. Therefore, the latent heat of melting was included, but the latent heat of vaporization was ignored.

Thermal conduction between particles was neglected: the particles were separated by about $1 \mu \mathrm{m}$ in Fig.3(a). Surface radiation from the upper semisphere surfaces to the environment was included. The initial temperature of the powder particles was set to $900 \mathrm{~K}$ to account for preheating.

The simulation results and more detailed discussions are presented in Section 5.1 and 5.2. 


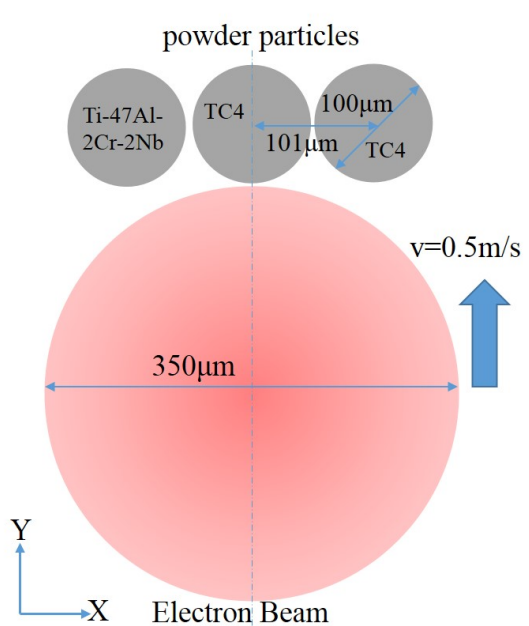

(a)

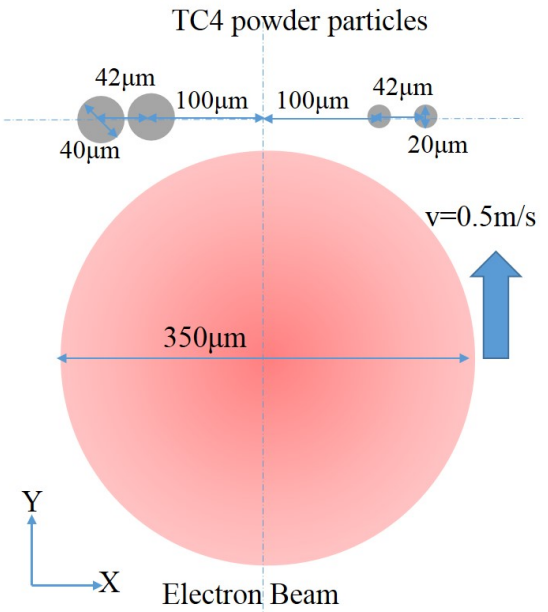

(b)

Figure 3: Schematic of a moving electron beam heating powder particles for (a) dissimilar particle compositions of the same size and (b) dissimilar particle sizes of the same composition.

\subsection{Effective thermal conductivity of the powder bed}

In order to pass a reasonable effective thermal conductivity of the original powder bed to the macro-scale model described in Section 4, we perform a simplified steady-state heat transfer simulation (3-Dimensional) shown in Fig.4 (a). Since the mixture ratio by volume of the two types of powder is around 1:1, we can expect a well-mixed bed of contacting particles of dissimilar compositions. Thus, the model contains two contacting particles, but only a half of each particle is constructed to reduce the computation cost. The radius of both particles is $R=0.05 \mathrm{~mm}$. The contact region is set to be a circle with a radius of $0.003 \mathrm{~mm}$. The temperature of the right boundary is set to $T_{1}=900 \mathrm{~K}$, and the left to $T_{2}=1000 \mathrm{~K}$. Surface radiation is neglected; this simplification is justified below. By calculating the total heat flux, $Q_{t}$, caused by the overall temperature gradient $\left(T_{X}=\frac{T_{2}-T_{1}}{2 R}=\frac{1000 \mathrm{~K}-900 \mathrm{~K}}{0.1 \mathrm{~mm}}=10^{6} \mathrm{~K} / \mathrm{m}\right)$, we can further evaluate the effective thermal conductivity as,

$$
\lambda_{p}=\frac{Q_{t}}{\pi R^{2} \times T_{X}}
$$

Previous studies evaluated the effective contact thermal conductivity systematically [17] [18]. Complex structures consisting of several particles in 
Table 2: Material parameters [15][16]

\begin{tabular}{llll}
\hline & Ti-6Al-4V & Ti-47Al-2Cr-2Nb & mixed $(3: 2)$ \\
\hline density $\left(\mathrm{kg} / \mathrm{m}^{3}\right)$ & 4400 & 3636 & 4100 \\
thermal conductivity $(\mathrm{W} /(\mathrm{m} \cdot \mathrm{K}))$ & 15 & 11 & 13.4 \\
specific heat $(\mathrm{J} /(\mathrm{kg} \cdot \mathrm{K}))$ & 700 & 727 & 710 \\
melting latent heat $(\mathrm{J} / \mathrm{kg})$ & 286000 & 377000 & 322000 \\
solidus temperature $(\mathrm{K})$ & 1878 & 1764 & 1764 \\
liquidus temperature $(\mathrm{K})$ & 1928 & 1785 & 1928 \\
\hline
\end{tabular}

height were considered, and the influence of the contact angle and contact size was investigated. In this study, the powder layer is no more than two powder particles thick; thus, there is no need to consider the influence of complex structures. Moreover, our proposed numerical model assigns different materials to the particles, which has not been considered by previous studies.

The simulation results are shown in Fig.4 (b), (c) and (d). The temperature changes sharply near the contact region, and the temperature in the remaining regions is almost uniform, as in the theoretical analysis [18]. The temperature gradient near the contact region is not symmetric due to different material properties on each side. Evident from Fig.4 (c) is that the heat flux intensity is distinctly larger near the contact region due to the higher temperature gradient. We integrate the heat flux on the boundary surface (see Fig.4 (d)) to get the total heat flux, $Q_{t}=8.17 \times 10^{-3} \mathrm{~W}$. The effective thermal conductivity is calculated to be $1.04 \mathrm{~W} /(\mathrm{m} \mathrm{K})$, according to Eq. 7 .

We justify neglecting surface radiation by theoretically approximating its influence on the effective thermal conductivity. The maximum thermal energy transferred through surface radiation is

$$
q_{\text {radiation }}=\sigma\left(T_{2}^{4}-T_{1}^{4}\right) \times 2 \pi R^{2}
$$

where $\sigma$ is the Boltzmann constant $\left(5.67 \times 10^{-8} \mathrm{~W} /\left(\mathrm{K}^{4} \mathrm{~m}^{2}\right)\right)$.

The maximum effective thermal conductivity due to surface radiation is

$$
\lambda_{p-\text { radiation }}=\frac{q_{\text {radiation }}}{\pi R^{2} \times T_{X}}=\frac{2 \sigma\left(T_{2}^{4}-T_{1}^{4}\right)}{\left(T_{2}-T_{1}\right) / 2 R}
$$

Eq. 9 shows that the maximum effective thermal conductivity due to 


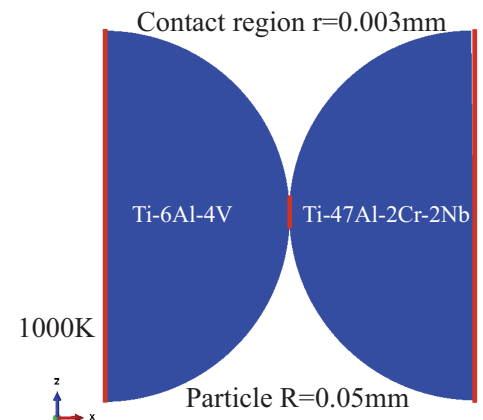

(a)

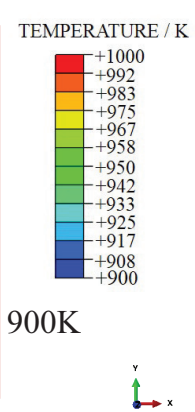

$\stackrel{\leftrightarrow}{a}$

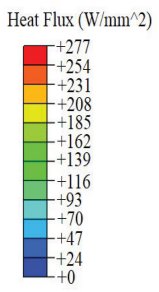

$\stackrel{\mathrm{t}}{\rightarrow} \times$

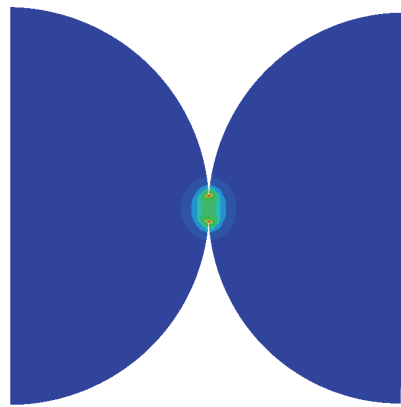

(c)

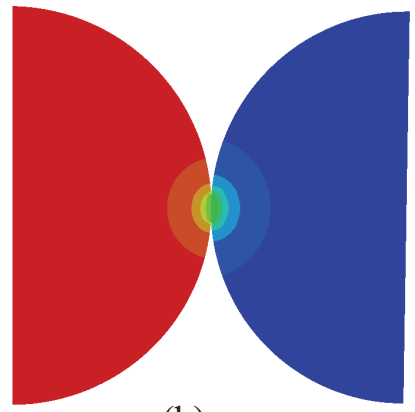

(b)

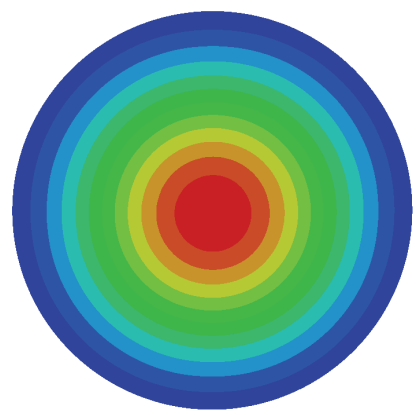

(d)

Figure 4: (a) Schematic of the meso-scale model for evaluating the effective thermal conductivity of the original powder bed. (b) Simulated temperature field and (c) heat flux distribution in the XY plane change sharply near the contact region. (d) Simulated heat flux distribution in the YZ cross section is used to calculate the effective thermal conductivity.

surface radiation largely depends on the temperature. For example, when $T_{2}=1000 \mathrm{~K}$ and $T_{1}=900 \mathrm{~K}$, the maximum effective thermal conductivity due to surface radiation is approximated as $0.04 \mathrm{~W} /(\mathrm{m} \mathrm{K})$, which is two orders smaller than the effective thermal conductivity due to contact. Even with $T_{2}=2000 \mathrm{~K}$ and $T_{1}=900 \mathrm{~K}, \lambda_{p-\text { radiation }}=0.16 \mathrm{~W} /(\mathrm{m} \mathrm{K})$ is still one order smaller. Therefore, it is reasonable to neglect the surface radiation when calculating the effective thermal conductivity.

\section{Macro-scale heat transfer model}

In the macro-scale model, we simplify the powder bed as an effective continuum as is common practice. However, the advance we propose is to track the coalescent state of the powder bed in the material model. Effective 
material properties are then derived based on the coalescing factor $F_{s} . F_{s}$ is defined in the range of 0 to 1 , where 0 and 1 denote the original powder bed state (no coalescence, see Fig.5(a)) and the bulk state (full coalescence, see Fig.5(c)), respectively. The coalescence is determined by the temperature history. The powder particles are partially melted and slightly sintered (see Fig.5(b)) once the temperature goes above the solidus temperature. As the temperature rises, the powder particles will be sintered together more and more tightly. Once the liquidus temperature is reached, the powder particles are considered completely melted together forming a bulk material. It should be noted that the increase of the coalescing factor is irreversible. As the temperature decreases, the bulk state after full melting will stay dense and the coalesced powder particles will no longer be discrete as they were in the original powder bed state. Therefore, the relationship between the coalescing state and the temperature history can be captured as,

$$
F_{s}=\frac{T_{p}-T_{s}}{T_{l}-T_{s}}
$$

where $T_{p}, T_{s}$, and $T_{l}$ denote the historically peak temperature, the solidus temperature and the liquidus temperature, respectively. As discussed in Section 3.2, the effective thermal conductivity of powder layer is related to the contact area due to sintering. The effective thermal conductivity of the sintered powder bed is assumed to be linearly dependent on the sintering state variable as:

$$
\lambda=\lambda_{b} F_{s}+\lambda_{p}\left(1-F_{s}\right)
$$

where $\lambda_{b}$ and $\lambda_{p}$ denote the thermal conductivity of the bulk material of the powder and the effective thermal conductivity in the original powder bed state, respectively. The effective thermal conductivity of the original porous powder bed is obtained from the aforementioned meso-scale model in Section 3.2 .

The respective fabrication model (see Fig.9 (a)) is built employing the same experimental parameters as in [1] but with a smaller material domain. In this model, the scan path is a single track of $3.5 \mathrm{~mm}$ long (duration time for each scan is $7 \mathrm{~ms}$ ), and the time interval between each scan is $3 \mathrm{~ms}$. The material properties of the mixed powder bed and substrate are obtained by taking a weighted average of the properties of the two component materials, except that the solidus temperature is set to the lower value and the liquidus temperature is set to the higher value, as listed in Table.2. This ensures 


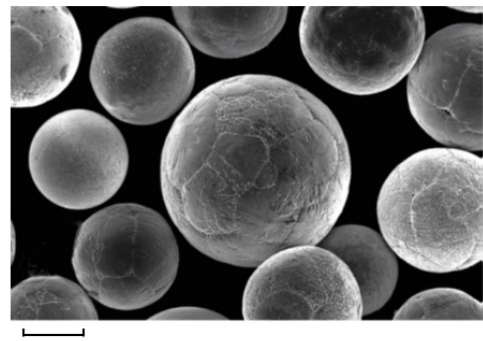

$50 \mu \mathrm{m}$

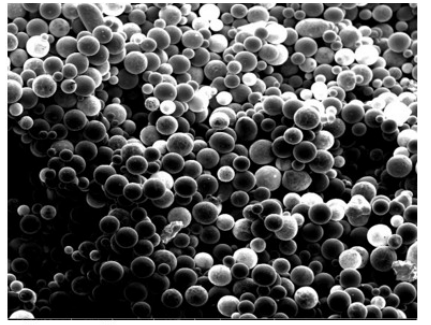

$500 \mu \mathrm{m}$

(a)

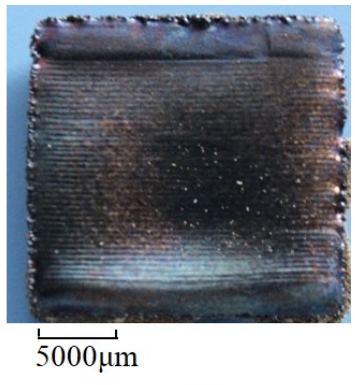

(c)

Figure 5: Different coalescent states of the powder bed. (a) original state $F_{s}=0$, (b) slightly sintered state, $0<F_{s}<1$ and (c) dense state, $F_{s}=1$.

that complete melting and solidification occur, even in regions locally rich in either alloy. Surface radiation of the top surface is considered.

\section{Multi-scale analysis of FGM fabrication process}

\subsection{Melting process of powder particles}

The simulated thermal evolution processes of powder particles of Ti-6Al$4 \mathrm{~V}$ and $\mathrm{Ti}-47 \mathrm{Al}-2 \mathrm{Cr}-2 \mathrm{Nb}$ are shown in Fig.6, where we label the liquidus temperature of Ti-6Al-4V and Ti-47Al-2Cr-2Nb as "TC4 melting" and "TiAl melting" in the legend. As is mentioned in Section 3.1, vaporization was ignored. The simulated peak temperatures in excess of the boiling temperatures of Ti-6Al-4V and Ti-47Al-2Cr-2Nb are a manifestation of this simplification.

Evident from Fig. 6 is that the TC4 particle in the middle gets fully melted first, followed by the Ti-47Al-2Cr-2Nb particle on the left, and finally the TC4 particle on the right. Comparing the results of 120W (see Fig.6 (a)) with those of 240W (see Fig.6 (b), (c) and (d)), we find that 120W is not high enough to fully melt all particles, but is able to fully melt particles along the central lines. This helps to sinter unmelted particles together during the first scan to prevent powder scattering under the following higher beam currents. At $360 \mathrm{~W}$, all the three particles become fully melted earlier.

The content distribution of Aluminum $(\mathrm{Al})$ was measured using an Electron Probe Micro-Analyzer (EPMA) as shown in Fig.7 [1]. The concentration of elemental Al changes gradually along the building direction in a layer-bylayer fashion. There are no observed pores or voids. Moreover, in each 


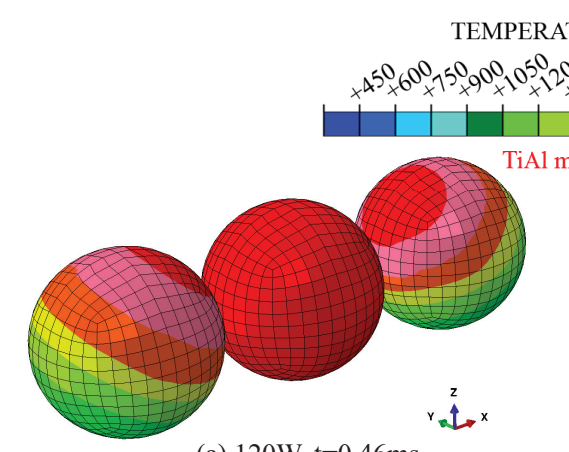

(a) $120 \mathrm{~W}, \mathrm{t}=0.46 \mathrm{~ms}$

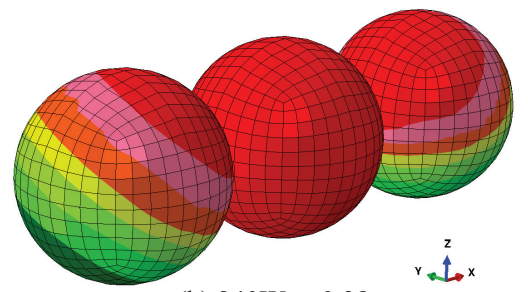

(b) $240 \mathrm{~W}, \mathrm{t}=0.38 \mathrm{~ms}$

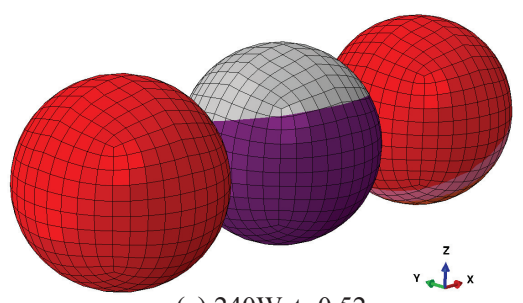

(c) $240 \mathrm{~W}, \mathrm{t}=0.52 \mathrm{~ms}$

gradient layer, the element is evenly distributed across a scale much smaller than the particle size. Thus, it can be concluded that all the powder particles were fully melted and evenly mixed. This matches with the prediction of complete melting under a power of $360 \mathrm{~W}$.

From the thermal analysis, we can make a suggestion for the fabrication parameter optimization as follows: first, apply a $2 \mathrm{~mA}$ electron beam for the first scan to fully melt some particles to prevent powder scattering, then to increase the beam current to $4 \mathrm{~mA}$ or $6 \mathrm{~mA}$ for the second scan to melt and coalesce all the particles. That is, we may get rid of the third scan in experiments to improve the production rate.

It should be also noted that in Fig.6 (b) the TC4 particle in the middle 


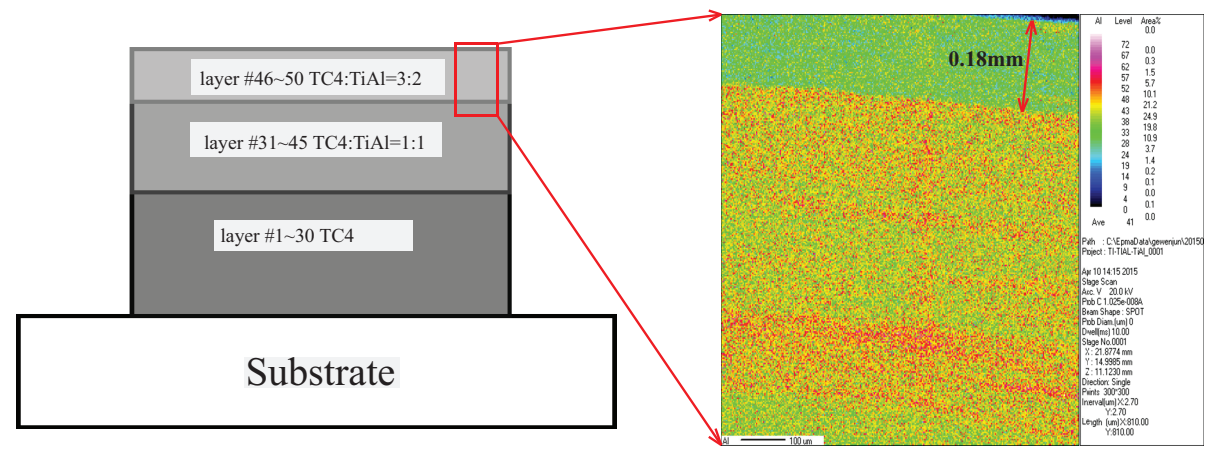

Figure 7: Schematic of the material gradient and the experimental measured distribution of $\mathrm{Al}[1]$.

becomes fully melted at $\mathrm{t}=0.38 \mathrm{~ms}$ even before the center of the electron beam arrives at the center of the particle at $\mathrm{t}=0.45 \mathrm{~ms}$. Thus, a more sophisticated model that captures the influence of phase changes on the heat source model may be required to fully understand the process.

\subsection{Effect of particle size}

The effect of the particle size on the melting and coalescing process was investigated by conducting the simulation case schematically shown in Fig.3 (b). The simulation results for powers of $120 \mathrm{~W}$ and $360 \mathrm{~W}$ are shown in Fig.8. As shown in Fig.8 (a) and (b), under a power of 120W, the particles located $100 \mu \mathrm{m}$ away from the central line with the diameters of $20 \mu \mathrm{m}$ and $40 \mu \mathrm{m}$ become fully melted, whereas the $100 \mu \mathrm{m}$ diameter particles are not fully melted in Fig.6 (a). As shown in Fig.8 (c) and (d), under a power of $360 \mathrm{~W}$, the particles located $100 \mu \mathrm{m}$ away from the central line with the diameters of $20 \mu \mathrm{m}$ and $40 \mu \mathrm{m}$ become fully melted at $\mathrm{t}=0.26 \mathrm{~ms}$ and $\mathrm{t}=0.30 \mathrm{~ms}$, respectively. The comparison of Fig.6 (f), Fig.8 (c) and Fig.8 (d) illustrates that the smaller particles melt more quickly due to the higher area-volume ratio.

Moreover, it should be noted that even the smaller particles located $140 \mu \mathrm{m}$ away from the central line of the electron beam become fully melted under a power of $360 \mathrm{~W}$. Alternatively speaking, smaller particles are more likely to get melted even if the electron beam power is low and the distance from the electron beam is large. This also explains why the average powder size becomes larger after being reused for several times [19]. This may also partially explain that the fabrication parameters for fresh powder do not 
work for reused powder in EBM, while other reasons include oxidation and shape change of powder particles.

It is reasonable to further infer that smaller particles may have considerable influence on preventing the powder scattering by binding larger particles after a low power scan.

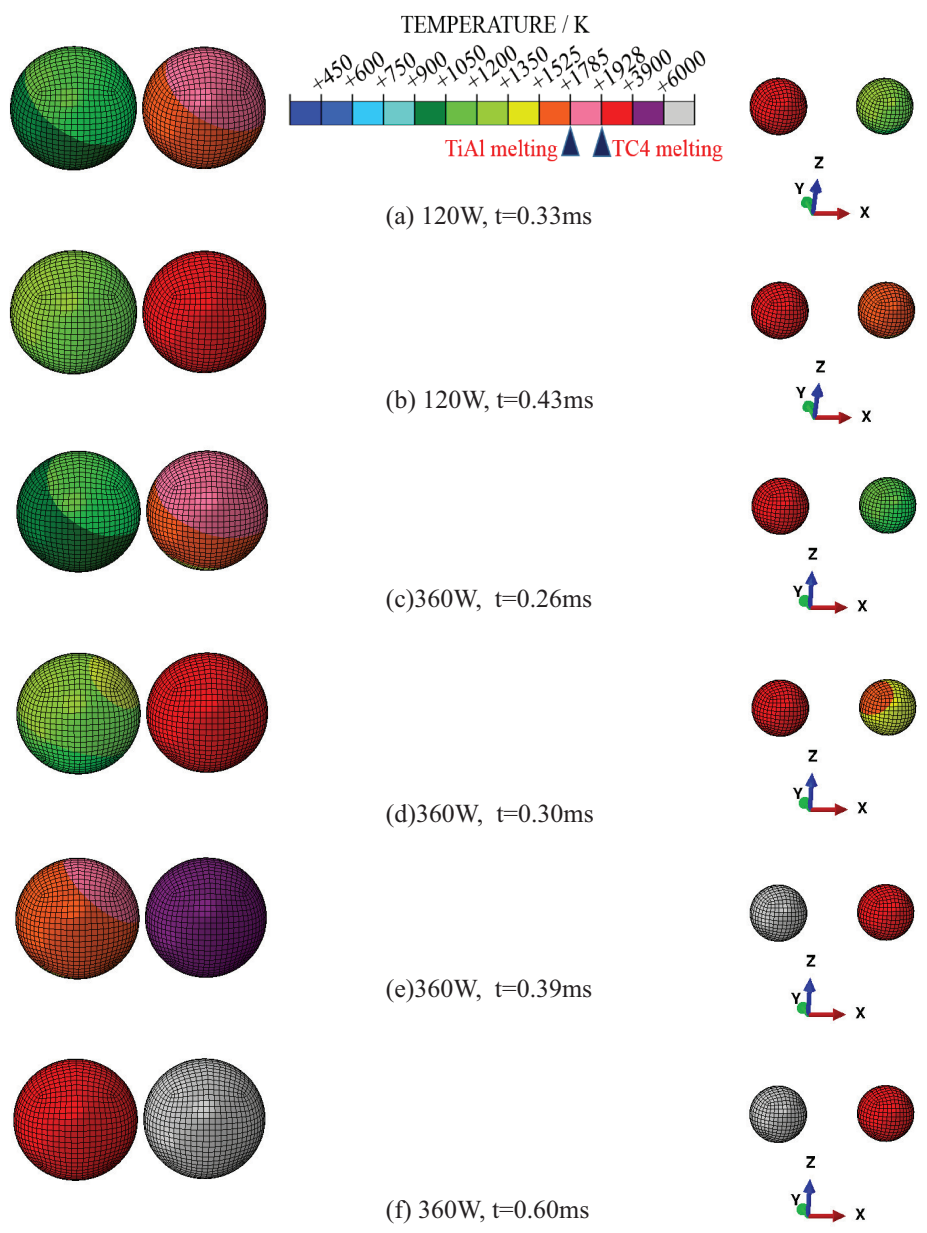

Figure 8: Thermal evolution of Ti-6Al-4V powder particles with various diameters showing the effect of size on particle heating.

\subsection{Molten pool size}

The macro-scale simulation results for the fabrication process are shown in Fig.9 (b), (c) and (d), respectively. The melting depth in the first scan 
is calculated to be $80 \mu \mathrm{m}$, which is smaller than the powder layer thickness. The melting depth in the second scan is calculated to be $120 \mu \mathrm{m}$. In the third scan, the depth of the melting region reaches $170 \mu \mathrm{m}$. The elemental distribution (see Fig.7) [1] reveals that the melting region of the final powder layer is $180 \mu \mathrm{m}$ thick. Thus, the simulation and experimental measurement are in good agreement.

The $10 \mu \mathrm{m}$ difference between the simulation and experiment is mainly attributed to the simplifications in the model. One major simplification is that only one single track rather than the actual process of multiple tracks was modeled. The heat accumulation due to the previous tracks was not incorporated, so the simulated melting depth is smaller than the real value. However, for the rapid prediction, this error about 5\% may be considered an acceptable trade-off.

A mesh convergence test was performed for all the simulations in this study to exclude the influence of mesh sensitivity. Relative change in the solution variables with a factor of 2 refine was under $5 \%$.

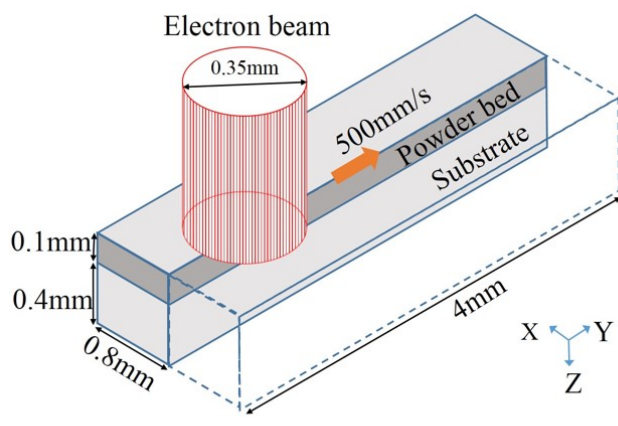

(a)

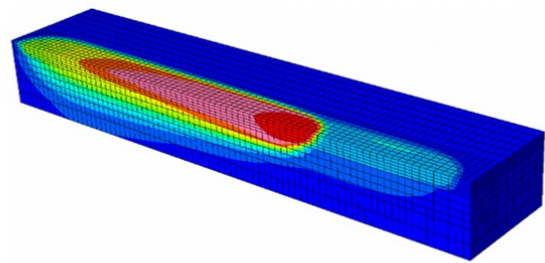

(c)

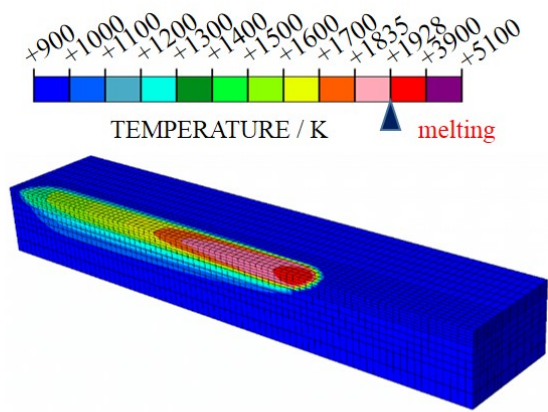

(b)

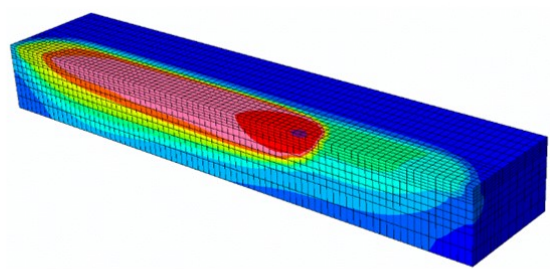

(d)

Figure 9: Macro-scale model. (a) schematic of the model, and simulation results at: (b) $\mathrm{t}=5 \mathrm{~ms}$, first scan $(2 \mathrm{~mA}),(\mathrm{c}) \mathrm{t}=15 \mathrm{~ms}$, second scan $(4 \mathrm{~mA})$ and $(\mathrm{d}) \mathrm{t}=25 \mathrm{~ms}$, third scan $(6 \mathrm{~mA})$ 


\section{Conclusion}

Derived from micro-scale electron-material interaction simulations, our proposed heat source model is capable of incorporating the material properties, electron beam properties and incidence angle. Thus, the heat source model is material dependent and experimental setup specific, thereby reducing uncertainty surrounding the fabrication process. This level of physical fidelity and the capacity to be used for process optimization is unprecedented.

The meso-scale model provides rapid prediction of the melting processes of mixed powder particles for FGMs. The simulations illustrate that a first scan with a power of $120 \mathrm{~W}$ only fully melts some particles on the scan lines and thus bond unmelted particles together, preventing powder scattering. A second scan with a power higher than $240 \mathrm{~W}$ then fully melts all particles including the particles between the scan lines, which is essential to the successful fabrication of FGMs. Furthermore, smaller particles fully melt more easily due to the high area-volume ratio, explaining the experimental observation that the average powder size increases with the reusing times. Thus, the meso-scale model can inform selection of proper fabrication parameters for different raw materials and powder size distributions.

In the macro-scale fabrication model, the effective material properties of the powder bed is adjusted by tracking the coalescent state, starting with an effective thermal conductivity derived from the meso-scale model. We performed a targeted simulation to reproduce the experimental fabrication process; the predicted size of the melting region agrees well with the experimental result. Along with the theoretical model [1] of the composition distribution in the FGMs manufactured by EBM, our macro-scale heat transfer model can help to design FGMs.

In the future, more research efforts will be devoted to simulating the evolution process of individual powder particles at the meso-scale, where the realistic heat source model will exert its advantages. Computationally efficient macro-scale fabrication models are required for industrial production. More information needs to be derived from meso-scale models and then incorporated into macro-scale models in order to provide more detailed and accurate predictions.

\section{Acknowledgments}

This research is sponsored by grants from National Institute of Standards and Technology (NIST) and Center for Hierarchical Materials Design 
(CHiMaD) under grant No. 70NANB13H194 and 70NANB14H012. Orion L. Kafka would like to thank the United States National Science Foundation (NSF) for their support through the NSF Graduate Research Fellowship Program (GRFP) under Financial award number DGE-1324585.

[1] W. Ge, F. Lin, C. Guo, Microstructure and mechanical property of electron beam selective melting ti6al4v/tial structural gradient material, in: 26th Annual International Symposium on Solid Freeform Fabrication, Austin, Texas.

[2] W. Ge, C. Guo, F. Lin, Microstructures of components synthesized via electron beam selective melting using blended pre-alloyed powders of ti6al4v and ti45al7nb, Rare Metal Materials and Engineering 44 (2015) $2623-2627$.

[3] C. Guo, W. Ge, F. Lin, Dual-material electron beam selective melting: Hardware development and validation studies, Engineering 1 (2015) 124.

[4] J. Smith, W. Xiong, J. Cao, W. K. Liu, Thermodynamically consistent microstructure prediction of additively manufactured materials, Computational Mechanics 57 (2016) 359-370.

[5] J. Smith, W. Xiong, W. Yan, S. Lin, P. Cheng, O. L. Kafka, G. J. Wagner, J. Cao, W. K. Liu, Linking process, structure, property, and performance for metal-based additive manufacturing: computational approaches with experimental support, Computational Mechanics 57 (2016) 583-610.

[6] S. Ghosh, J. Choi, Three-dimensional transient finite element analysis for residual stresses in the laser aided direct metal/material deposition process, Journal of Laser Applications 17 (2005) 144-158.

[7] B. Schoinochoritis, D. Chantzis, K. Salonitis, Simulation of metallic powder bed additive manufacturing processes with the finite element method: A critical review, Proceedings of the Institution of Mechanical Engineers, Part B: Journal of Engineering Manufacture (2015).

[8] W. Yan, W. Ge, J. Smith, G. Wagner, F. Lin, W. K. Liu, Towards highquality selective beam melting technologies: Modeling and experiments of single track formations, in: 26th Annual International Symposium on Solid Freeform Fabrication, Austin, Texas. 
[9] C. Körner, A. Bauereiß, E. Attar, Fundamental consolidation mechanisms during selective beam melting of powders, Modelling and Simulation in Materials Science and Engineering 21 (2013) 085011.

[10] S. A. Khairallah, A. T. Anderson, A. Rubenchik, W. E. King, Laser powder-bed fusion additive manufacturing: Physics of complex melt flow and formation mechanisms of pores, spatter, and denudation zones, Acta Materialia 108 (2016) 36-45.

[11] W. King, A. Anderson, R. Ferencz, N. Hodge, C. Kamath, S. Khairallah, Overview of modelling and simulation of metal powder bed fusion process at lawrence livermore national laboratory, Materials Science and Technology 31 (2015) 957-968.

[12] S. A. Khairallah, A. Anderson, Mesoscopic simulation model of selective laser melting of stainless steel powder, Journal of Materials Processing Technology 214 (2014) 2627-2636.

[13] W. Yan, J. Smith, W. Ge, F. Lin, W. K. Liu, Multiscale modeling of electron beam and substrate interaction: a new heat source model, Computational Mechanics 56 (2015) 265-276.

[14] D. B. Williams, C. B. Carter, Transmission electron microscopy: a textbook for materials science (2009).

[15] M. Jamshidinia, F. Kong, R. Kovacevic, Numerical modeling of heat distribution in the electron beam melting $($ R of ti-6al- $4 \mathrm{v}$, Journal of Manufacturing Science and Engineering 135 (2013) 061010.

[16] X. Xu, H. Chang, H. Kou, Z. Yang, J. Li, Simulation of temperature field distribution in melting tial alloy by pam process, International Journal of Innovative Technology and Exploring Engineering (IJITEE) 2 (2013) 31-35.

[17] W. Siu, S.-K. Lee, Effective conductivity computation of a packed bed using constriction resistance and contact angle effects, International journal of heat and mass transfer 43 (2000) 3917-3924.

[18] A. Gusarov, T. Laoui, L. Froyen, V. Titov, Contact thermal conductivity of a powder bed in selective laser sintering, International Journal of Heat and Mass Transfer 46 (2003) 1103-1109. 
518

519

520

[19] H. P. Tang, M. Qian, N. Liu, X. Z. Zhang, G. Y. Yang, J. Wang, Effect of powder reuse times on additive manufacturing of ti-6al-4v by selective electron beam melting, JOM 67 (2015) 555-563. 
Abstract: In this study, we develop a novel multi-scale heat transfer modeling framework to investigate the EBM process of fabricating FGMs. Our heat source model is based on micro-scale electron-material interaction simulations. It is capable of accounting for the material properties and electron beam properties that are dependent on experimental setup. The heat source model is utilized in the thermal evolution model of individual powder particles at the meso-scale to elucidate the melting and coalescing processes for mixed powder particles of different materials and different sizes. Another meso-scale simulation is conducted to evaluate the effective thermal conductivity of the original powder bed for the macro-scale model. A macro-scale heat transfer model is developed, in which the coalescence state is tracked to determine the effective material properties of the powder bed. Predictions of molten pool size are compared against published experimental results for validation.

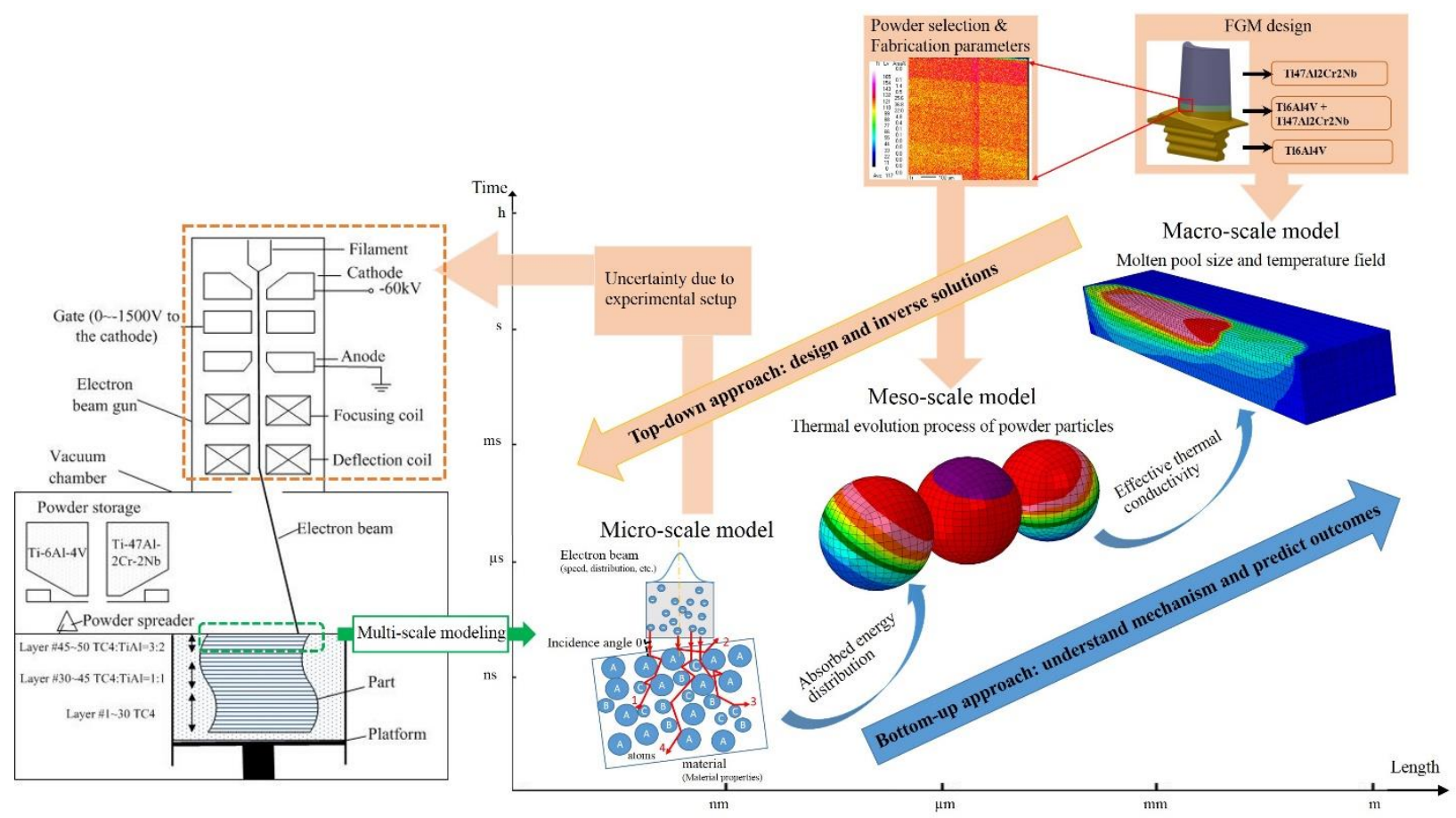

